\title{
Spatial and Temporal Packet Recovery Schemes for DVB-H Systems through IP-Relay Wireless Networks
}

\author{
Wen-Hsin Yang ${ }^{1,3}$, You-Chiun Wang ${ }^{1}$, Yu-Chee Tseng ${ }^{1,2}$, and Bao-Shuh P. Lin ${ }^{1,3}$ \\ ${ }^{1}$ Department of Computer Science, National Chiao-Tung University, Taiwan \\ ${ }^{2}$ Department of Information and Computer Engineering, Chung-Yuan Christian University, Taiwan \\ ${ }^{3}$ Information and Communications Research Labs, Industrial Technology Research Institute, Taiwan \\ Email: \{wenhsin, wangyc, yctseng\}@cs.nctu.edu.tw; bplin@itri.org.tw
}

\begin{abstract}
The DVB-H standard has been defined to provide digital video broadcast to mobile handheld devices. However, without a request channel, a handheld device may encounter data loss and thus it requires an additional recovery architecture to relieve this problem. In this paper, we follow the DVB-IPDC standard and propose combining a DVB-H system with an IPrelay wireless network. A handheld device losing DVB-H data can thus request the relay network for retransmission. Under this architecture, we identify two critical issues, group packet loss $(G P L)$ and broadcast data handover $(B D H)$. The GPL problem occurs when there are bursty requests for retransmissions of the same data with high spatial and temporal locality, causing network congestion. The BDH problem occurs when these requesting devices move out of their current serving cells. In this paper, we propose a bulk request recovery $(B R R)$ scheme to solve these problems by exploiting spatial and temporal locality of recovery requests. Our scheme can efficiently reduce duplicate requests and schedule retransmissions of lost packets, so both GPL and BDH problems are relieved. Simulations results are also presented to verify the effectiveness of our result.
\end{abstract}

Index Terms-broadcasting network, DVB-H, error recovery, scheduling, video broadcast, wireless network.

\section{INTRODUCTION}

The DVB-H (digital video broadcasting-handheld) standard [1], [2] has been proposed to support digital video broadcast for mobile handheld devices (HDs). It adopts a mandatory time slicing technology and an optional MPE-FEC (multi-protocol encapsulation-forward error correction) method to broadcast multimedia contents to HDs. With time slicing, datagrams are transmitted as data bursts in small time slots. On the other hand, MPE-FEC can help tolerate some degrees of noises.

However, HDs are more vulnerable to packet loss due to their hardware constraints and high mobility [3]. There are two possible solutions. One solution is to retransmit lost packets using the DVB-H return channel. However, this may complicate the design of the DVB-H server because it will need to take care of both broadcast and retransmissions. Not only retransmissions need to share the bandwidth with regular broadcast [4], but also the return channel is relatively small. Another solution is DVB-IPDC (IP datacast over DVB-H) [5], which requires to provide a separate IP-based wireless network to offer a bi-directional interactive communication channel to

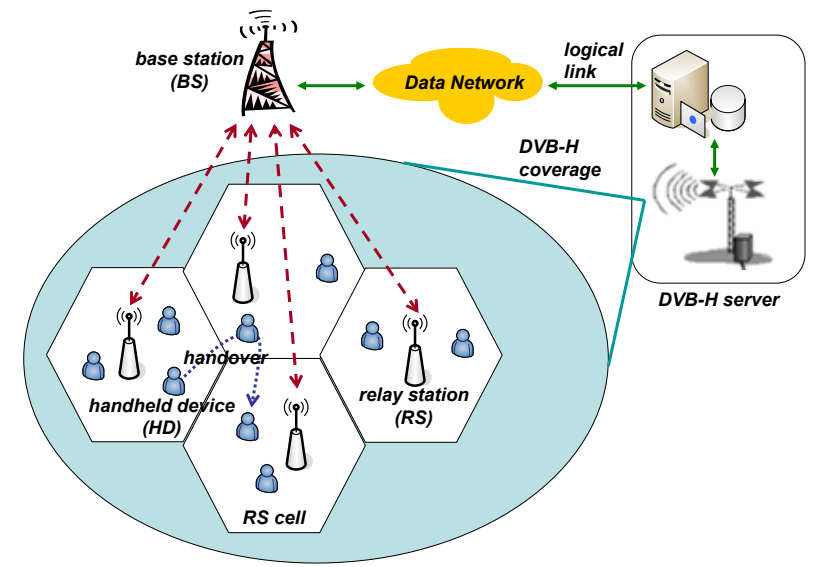

Fig. 1: The integrated architecture of a DVB-H system and an IP-relay wireless network.

request and send lost packets. This solution is more feasible because the broadcast and retransmissions are separated.

In this paper, we adopt the DVB-IPDC solution with a broadband wireless channel such as WiMAX [6], [7] to support packet recovery, as shown in Fig. 1. HDs will roam inside the coverage of both DVB-H and WiMAX networks. The DVB-H server continuously broadcasts digital video to HDs. However, since HDs are more vulnerable to broadcast packet loss and corruption, HDs can request nearby relay stations $(R S s)$ to retransmit lost packets. Under this architecture, we identify two critical issues, group packet loss (GPL) and broadcast data handover $(B D H)$. The GPL problem occurs when there are bursty requests for retransmissions of the same data with high spatial and temporal locality. Thus, some RSs may be badly congested by incoming requests and outgoing retransmissions of lost packets. Such phenomena may even cause many packets to miss their deadlines. The BDH problem occurs when the requesting HDs handover to neighboring cells after making their requests. Duplicate requests and retransmissions thus may further congest the relay network.

To solve these problems, we should inhibit HDs from submitting duplicate requests and properly deliver the lost 
packets. We observe that recovery requests may exhibit some spatial and temporal locality. In the space domain, neighboring HDs may submit similar requests because they are interfered by the same noises. Moreover, the handover behavior of the HDs will extend this spatial feature to neighboring cells. In the time domain, HDs in the same cell may lose a similar sequence of packets because interference sources often exist for a period of time. Pre-fetch and pre-downloading are potential solutions. Therefore, there is a large space to merge redundant requests and retransmissions to improve efficiency.

In this paper, we propose a bulk request recovery $(B R R)$ scheme by considering spatial and temporal locality of recovery requests. Each RS will collect HDs' requests for a short period of time, and then broadcast a group-acknowledgement $(g A C K)$ to announce what requests have already been received by the RS. This gACK exploits spatial and temporal locality to inhibit HDs from submitting duplicate requests, in both space and time domains. Then, the RS schedules and packs these requests into a bulk request and sends to the base station $(B S)$, which will in turn forward them to the DVB$\mathrm{H}$ server. To exploit spatial locality, the BS will use broadcast to send these lost packets to neighboring RSs. In this way, the overheads are reduced. To exploit temporal locality, the BS will pre-fetch some correlated packets in the time domain from the DVB-H server to reduce the downloading latency. Our major contributions are two-fold. First, we address the GPL and BDH problems in an integrated architecture of DVB$\mathrm{H}$ systems and IP-relay wireless networks. Second, we develop a novel BRR scheme to solve these problems by exploiting spatial and temporal locality of recovery requests and packet retransmissions. Simulation results have shown that BRR can efficiently reduce the amount of packet retransmissions.

In the literature, several studies have considered combining DVB-H systems with wireless networks. Automatic configuration of an IP-based interworking model among WLAN, $3 \mathrm{G}$, and DVB-H is addressed in [8]. But it does not discuss the retransmission issue. Reference [9] proposes a multicast protocol to recover DVB-H loss with IEEE 802.11 WLANs. The work [10] discusses how to support mobile TV services by combining DVB-H and 3G networks, and evaluates the impact of delivering parity data through $3 \mathrm{G}$ networks. Reference [11] also considers a hybrid system of DVB-H and cellular networks, and discusses how to retransmit the corrupted data through the cellular network. However, all these works do not consider the GPL and BDH problems discussed in this paper.

The rest of this paper is organized as follows. Section II presents our system architecture. Section III proposes our BRR scheme. Section IV gives our simulations results. Conclusions are drawn in Section V.

\section{INTEGRATED ARChIteCtURE OF DVB-H Systems AND IP-RELAY WIRELESS NETWORKS}

Fig. 1 shows the integrated architecture of a DVB-H system and an IP-relay wireless network. Each HD is equipped with a DVB-H receiver and a wireless interface. Here, we adopt WiMAX as the IP-relay network, which consists of multiple

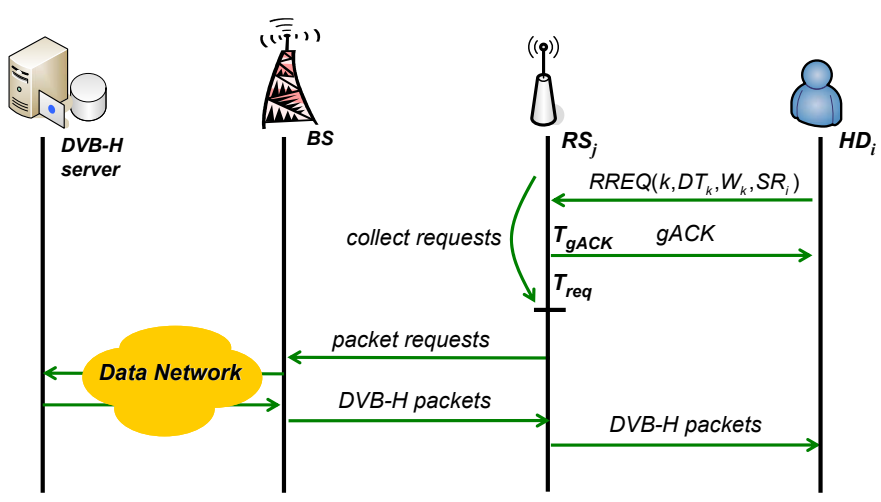

Fig. 2: The proposed BRR scheme.

BSs that can communicate with the DVB-H server through a data network. Each BS can support multiple RSs. HDs will continuously receive the DVB-H broadcast data. When an HD experiences packet loss, it can request a neighboring RS to resend these lost packets. Then, the BS will collect these requests and query the DVB-H server for the lost packets. Finally, the BS will transmit them to the requesting HDs through the corresponding RSs.

\section{The Bulk Request Recovery (BRR) Scheme}

As pointed out earlier, recovery requests from HDs may exhibit some spatial and temporal locality. Neighboring HDs will likely lose the similar sequences of DVB-H packets since they may experience the same noises over the same durations. As these HDs handover to other cells, neighboring RSs may also observe the same locality. With these observations, we propose our BRR scheme below (refer to Fig. 2).

1) Each $\mathrm{HD}_{i}$ has three states, as shown in Fig. 3(a). Initially, $\mathrm{HD}_{i}$ is in the listening state, where it does nothing but continuously receives broadcast packets from the DVB-H server. However, when $\mathrm{HD}_{i}$ detects the loss of any DVB-H packet, it switches to the submitting state.

2) In the submitting state, $\mathrm{HD}_{i}$ maintains a buffer $B_{i}$ to store the indices of the lost packets and then submits recovery requests according to $B_{i}$ 's content. Specifically, suppose that $\mathrm{HD}_{i}$ loses a DVB-H packet $p_{k}$, it will store the index of $p_{k}$ (i.e., $k$ ) in $B_{i}$ and then unicast a recovery request $R R E Q\left(k, D T_{k}, W_{k}, S R_{i}\right)$ to its associating $\mathrm{RS}_{j}$, where $D T_{k}$ is $p_{k}$ 's due time, $W_{k}$ is $p_{k}$ 's weight, and $S R_{i}$ is $\mathrm{HD}_{i}$ 's subscriber right. Here, we use $D T_{k}$, $W_{k}$, and $S R_{i}$ to reflect the priority of packet $p_{k}$ to $\mathrm{HD}_{i}$. (For example, $W_{k}$ may range from 1 to 4 and $S R_{i}$ may range from 1 to 3 . A higher value means a higher priority.) After confirming that $\mathrm{RS}_{j}$ has received the request (by receiving a lower-layer ACK message from $\mathrm{RS}_{j}$ ), $\mathrm{HD}_{i}$ will remove the index of $p_{k}$ from $B_{i}$. $\mathrm{HD}_{i}$ will repeat the above operation if there are other entries in $B_{i}$. However, two cases will make $\mathrm{HD}_{i}$ leave the submitting state: First, a timer $T_{r e q}$ expires. Second, $\mathrm{RS}_{j}$ does not reply after several tries of submissions. We will discuss these two cases later in step 6 . 


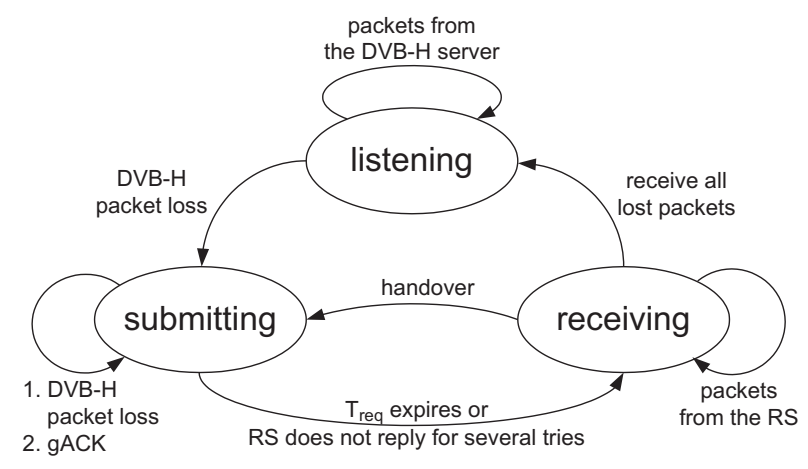

(a) the case of each $\mathrm{HD}_{i}$

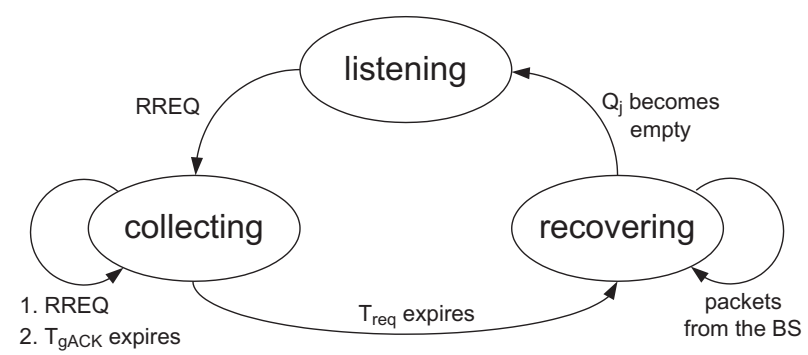

(b) the case of each $\mathrm{RS}_{j}$

Fig. 3: State transition diagrams of HDs and RSs.

3) Each $\mathrm{RS}_{j}$ also has three states, as shown in Fig. 3(b). Initially, $\mathrm{RS}_{j}$ is in the listening state, where it will continuously listen if there is any recovery request from any $\mathrm{HD}$. If so, $\mathrm{RS}_{j}$ will switch to the collecting state.

4) In the collecting state, $\mathrm{RS}_{j}$ sets up a timer $T_{g A C K}$ and maintains a counter $c_{j}$ to record the number of recovery requests that it has received. Then, two operations will be conducted:

a) $\mathrm{RS}_{j}$ keeps on collecting recovery requests from HDs. These requests are maintained in a request queue $Q_{j}$, in which each entry consists of fivetuple $\left(k, \min T_{k}, N_{k}, W_{k}, P F_{k}\right)$, where $N_{k}$ is the number of HDs that have requested packet $p_{k}$ and $P F_{k}$ is the expected profit of rebroadcasting $p_{k}$. Supposing that devices $\mathrm{HD}_{\alpha_{1}}, \mathrm{HD}_{\alpha_{2}}, \cdots$, and $\mathrm{HD}_{\alpha_{N_{k}}}$ have requested $p_{k}$ with due time $D T_{k}^{\alpha_{1}}$, $D T_{k_{2}}^{\alpha_{2}}, \cdots$, and $D T_{k}^{\alpha_{N_{k}}}$, we set $\min D T_{k}=$ $\min _{i=1}^{N_{k}}\left\{D T_{k}^{\alpha_{i}}\right\}$. Also, one way to define the profit is $P F_{k}=W_{k} \times \sum_{i=1}^{N_{k}} S R_{\alpha_{i}}$.

b) When $c_{j}$ exceeds a predefined threshold $\delta_{g A C K}$ or timer $T_{g A C K}$ expires, $\mathrm{RS}_{j}$ will set up another timer $T_{\text {req }}$ and broadcast a group-acknowledgement $g A C K\left(\left(\beta_{1}, \beta_{2}, \cdots, \beta_{\left|Q_{j}\right|}\right),\left(\beta_{\left|Q_{j}\right|+1}, \beta_{\left|Q_{j}\right|+2}, \cdots\right.\right.$, $\left.\left.\beta_{\left|Q_{j}\right|+\gamma}\right), T_{r e q}\right)$, where $\left(\beta_{1}, \beta_{2}, \cdots, \beta_{\left|Q_{j}\right|}\right)$ are the indices of the lost packets recorded in $Q_{j}$, $\left(\beta_{\left|Q_{j}\right|+1}, \beta_{\left|Q_{j}\right|+2}, \cdots, \beta_{\left|Q_{j}\right|+\gamma}\right)$ are the indices of the "predicted" lost packets, and $T_{r e q}$ is the expected time that $\mathrm{RS}_{j}$ will stop collecting the recovery requests sent from $\mathrm{HDs}$. $\mathrm{RS}_{j}$ will also create the corresponding entries of these predicted packet indices $\beta_{\left|Q_{j}\right|+1}, \beta_{\left|Q_{j}\right|+2}, \cdots$, and $\beta_{\left|Q_{j}\right|+\gamma}$ in $Q_{j}$. The due time of these new entries may be approximated by a linear interpolation. One way to define the weight and profit values of these new entries is to take the average weight and profit of original entries.

When timer $T_{r e q}$ expires, $\mathrm{RS}_{j}$ will switch to the recovering state.

5) On receiving $g A C K$, each $\mathrm{HD}_{i}$ in the submitting state removes those packet indices that appear in $g A C K$ from its buffer $B_{i}$. Then, before timer $T_{r e q}$ expires, HDs will still submit their requests to $\mathrm{RS}_{j}$. Here, we can exploit spatial and temporal locality to reduce the number of submitting recovery requests by $g A C K$. In the space domain, $g A C K$ will inhibit HDs from submitting duplicate requests. When one HD successfully submits the request of lost packet $p_{k}$, other neighboring HDs that also lose $p_{k}$ but have not submitted the corresponding requests yet will cancel their submissions when they hear $g A C K$. In the time domain, we expect HDs will lose a continuous sequence of packets in the near future if they have already done so. For example, supposing that a continuous sequence of packet indices $\left(\beta_{\left|Q_{j}\right|}-k, \beta_{\left|Q_{j}\right|}-k+1, \cdots, \beta_{\left|Q_{j}\right|}\right)$ have appeared in $Q_{j}, \mathrm{RS}_{j}$ will add the following packet indices $\left(\beta_{\left|Q_{j}\right|}+1, \beta_{\left|Q_{j}\right|}+2, \cdots, \beta_{\left|Q_{j}\right|}+\gamma\right)$ in $g A C K$, where $\gamma<k$. In this way, HDs will cancel some of their future submissions if their requests possess temporal locality.

6) For each $\mathrm{HD}_{i}$ in the submitting state, after timer $T_{r e q}$ expires, it changes to the receiving state to wait for the retransmission of lost DVB-H packets from $\mathrm{RS}_{j}$. In this case, if there still remains packet indices in its buffer $B_{i}, \mathrm{HD}_{i}$ clears $B_{i}$ since these packets may exceed their deadlines. However, in the case that an $\mathrm{HD}_{i}$ does not hear $g A C K$ in step 5, it will still submit its requests to $\mathrm{RS}_{j}$ even though timer $T_{r e q}$ has expired. In this case, $\mathrm{RS}_{j}$ will not confirm the receipt of these requests. After several tries of failed submissions, $\mathrm{HD}_{i}$ will realize that timer $T_{\text {req }}$ has expired and thus it will switch to the receiving state. $A n \mathrm{HD}_{i}$ in the receiving state will continue receiving DVB-H packets from the $\mathrm{RS}$, and will go back to the listening state when it has received all its lost DVB-H packets. Note that it is possible for $\mathrm{HD}_{i}$ to go back to the submitting state when a handover event occurs, and we will discuss this later.

7) For each $\mathrm{RS}_{j}$ in the collecting state, after timer $T_{r e q}$ expires, it will switch to the recovering state and clear its counter $c_{j}$. Then, two operations will be conducted:

a) $\mathrm{RS}_{j}$ sorts the entries of $Q_{j}$ in an increasing order of packets' due time $D T_{k}$. When two packets have the same due time, we use their profits $P F_{k}$ to break the tie. The packet with a larger profit will have a higher priority. Fig. 4 gives an example.

b) $\mathrm{RS}_{j}$ dequeues $m$ packets from $Q_{j}$ at a time and transmits them to the BS. After collecting packet 


\begin{tabular}{|c|c|c|c|c|c|c|c|c|c|}
\hline \multirow[b]{2}{*}{$p_{k}$} & \multicolumn{4}{|c|}{ head of $Q_{j}$} & \multicolumn{5}{|c|}{ head of $Q_{j}$} \\
\hline & 5 & 4 & 3 & 1 & $p_{k}$ & 4 & 5 & 3 & 1 \\
\hline$D T_{k}$ & 1009 & 1009 & 1007 & 1005 & $D T_{k}$ & 1009 & 1009 & 1007 & 1005 \\
\hline$N_{k}$ & 2 & 1 & 1 & 1 & $N_{k}$ & 1 & 2 & 1 & 1 \\
\hline$W_{k}$ & 3 & 4 & 2 & 4 & $W_{k}$ & 4 & 3 & 2 & 4 \\
\hline$P F_{k}$ & 15 & 12 & 2 & 12 & $P F_{k}$ & 12 & 15 & 2 & 12 \\
\hline & 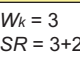 & $\begin{array}{l}W_{k}=4 \\
S R=3\end{array}$ & $\begin{array}{l}W_{k}=2 \\
S R=1\end{array}$ & $\begin{array}{l}W_{k}=4 \\
S R=3\end{array}$ & & $\begin{array}{l}W_{k}=4 \\
S R=3\end{array}$ & $\begin{array}{l}\text { k }=3 \\
S R=3+2\end{array}$ & $\begin{array}{l}W_{k}=2 \\
S R=1\end{array}$ & $\begin{array}{l}W_{k}=4 \\
S R=3\end{array}$ \\
\hline
\end{tabular}

Fig. 4: An example of scheduling the request queue $Q_{j}$.

requests from RSs, the BS will query the DVB$\mathrm{H}$ server for the lost packets, through the data network. Then, the BS will transmit these DVB$\mathrm{H}$ packets to the requesting RSs. Here, we can also exploit spatial and temporal locality to reduce the transmission amount and latency of DVB-H packets from the BS to RSs. In the space domain, we can first group neighboring RSs into clusters. Then, for each cluster, the BS will broadcast their requesting DVB-H packets together. Since packet requests of neighboring cells may overlap with each other (due to spatial locality), the amount of DVB-H packets broadcasted by the BS can thus be reduced. In the time domain, when the BS finds that RSs request a continuous sequence of DVB$\mathrm{H}$ packets, it can pre-fetch the following packets from the DVB-H server. In this way, the latency to reply packets to RSs can be reduced.

$\mathrm{RS}_{j}$ will repeat operation (b) until $Q_{j}$ becomes empty. In this case, $\mathrm{RS}_{j}$ will go back to the listening state.

We then discuss the handover issue. When an $\mathrm{HD}_{i}$ submits its recovery requests to an $\mathrm{RS}_{j}$ but later handovers to another $\mathrm{RS}_{l}, \mathrm{HD}_{i}$ will switch to the submitting state and try to resubmit its requests to $\mathrm{RS}_{l}$. However, due to spatial locality, some HDs in $\mathrm{RS}_{l}$ 's cell may have already submitted the same requests. Thus, by step $5, \mathrm{HD}_{i}$ would cancel its submissions when it hears $g A C K$ sent by $\mathrm{RS}_{l}$. In addition, in step 7, the BS will broadcast the requesting DVB-H packets to neighboring RSs. This also guarantees that HDs can receive their requesting packets when they handover to adjacent cells.

Fig. 5 gives an example of the BRR scheme, where each RS dequeues two packets at one time (i.e., $m=2$ ). Suppose that $\mathrm{HD}_{3}$ handovers from $\mathrm{RS}_{1}$ to $\mathrm{RS}_{2}$ after submitting a recovery request of packet $p_{1}$. In Fig. 5(a), after $\mathrm{RS}_{2}$ broadcasts $g A C K, \mathrm{HD}_{3}$ will cancel its submission because $p_{1}$ has already been requested by $\mathrm{HD}_{5}$. Then, $\mathrm{RS}_{1}$ and $\mathrm{RS}_{2}$ will schedule their request queues and send the requests $\operatorname{RREQ}(2,1)$ and $R R E Q(1,4)$ to the $\mathrm{BS}$, respectively. After obtaining packets $p_{1}, p_{2}$, and $p_{4}$ from the DVB-H server, the BS will broadcast them to $\mathrm{RS}_{1}$ and $\mathrm{RS}_{2}$, as shown in Fig. 5(b). Since $p_{1}$ is requested by both $\mathrm{RS}_{1}$ and $\mathrm{RS}_{2}$, we can avoid duplicate retransmissions and thus reduce the amount of DVB-H packets transmitted by the BS.

We finally comment on the timing for an $\mathrm{RS}_{j}$ to broadcast $g A C K$. Recall that $\mathrm{RS}_{j}$ will broadcast $g A C K$ when either its

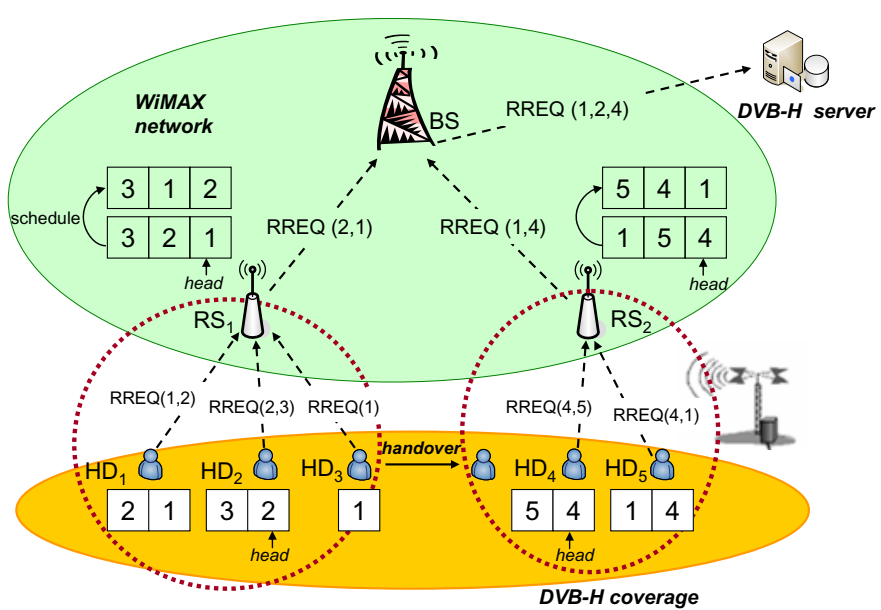

(a) submit recovery requests

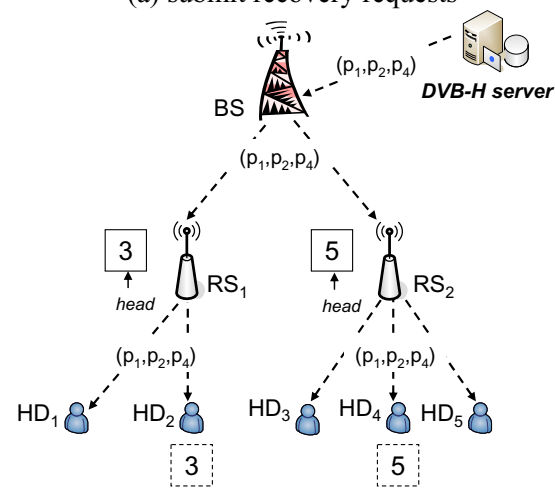

(b) obtain lost packets

Fig. 5: An example of the BRR scheme.

timer $T_{g A C K}$ expires or $c_{j}>\delta_{g A C K}$. There are two possible scenarios. In the first scenario, all serving HDs submit their requests very sparsely. In this case, it means that the recovery requests exhibit very less spatial and temporal locality, so $\mathrm{RS}_{j}$ prefers broadcasting $g A C K$ after collecting more requests. Since $c_{j}$ may be always less than $\delta_{g A C K}$ before $T_{g A C K}$ expires, $\mathrm{RS}_{j}$ can thus collect more requests at the expiration of $T_{g A C K}$. In the second scenario, some HDs submit a continuous sequence of requests (i.e., high temporal locality) or many HDs submit their requests in a short duration (i.e., high spatial locality). In this case, since $c_{j}$ will exceed $\delta_{g A C K}$ before $T_{g A C K}$ expires, $\mathrm{RS}_{j}$ can thus fast broadcast $g A C K$ to reflect the spatial and temporal locality of recovery requests.

\section{PERFormance EVAluation}

In this section, we verify the effectiveness of our BRR scheme by simulations. We measure the average amount of packets delivered from an RS to its serving HDs, under different distributions of DVB-H packet loss $\mathcal{E}$ and locality features $\mathcal{L}$ of recovery requests. Specifically, the distribution $\mathcal{E}$ indicates the necessity of submitting recovery requests by HDs, while the distribution $\mathcal{L}$ indicates the inhibited submissions of recovery requests after the RS broadcasts $g A C K$. In the simulations, we assume that $\mathcal{E}$ and $\mathcal{L}$ are normal distributions 
and independent with each other. We set $\mathcal{E}$ and $\mathcal{L}$ as follows: (1) packet loss (skew left): $\mathcal{E}\left(x=0 \sim T, \mu=\frac{1}{4} T, \sigma=\frac{1}{5} T\right)$, (2) packet loss (balance): $\mathcal{E}\left(x=0 \sim T, \mu=\frac{1}{2} T, \sigma=\frac{1}{5} T\right)$, (3) packet loss (skew right): $\mathcal{E}\left(x=0 \sim T, \mu=\frac{3}{4} T, \sigma=\frac{1}{5} T\right)$, (4) locality (middle): $\mathcal{L}\left(x=0 \sim T, \mu=\frac{1}{2} T, \sigma=\frac{1}{6} T\right)$, (5) locality (late): $\mathcal{L}\left(x=0 \sim T, \mu=\frac{3}{4} T, \sigma=\frac{1}{6} T\right)$, where $x$, $\mu$, and $\sigma$ are variable, mean value, and standard deviation of the distribution, respectively, and $T=T_{g A C K}+T_{r e q}$. For the distribution $\mathcal{E}$, three cases of skew left, balance, and skew right indicate that the most packet loss occur at $0.25 T, 0.5 T$, and $0.75 T$, respectively. For the distribution $\mathcal{L}$, two cases of middle and late indicate that the most locality of recovery requests occur at $0.5 T$ and $0.75 T$, respectively.

Fig. 6 shows the transmission amount of lost packets under different distributions of $\mathcal{E}$ and $\mathcal{L}$. When BRR is not adopted, HDs will sequentially submit their recovery requests to the RS (even though the requests have already been submitted by other HDs) and the RS will then unicast the lost packets to the requesting HDs. The length of $T_{g A C K}$ is set to 0 , $0.1 T, 0.2 T, \cdots$, and $T$. In Fig. 6 , we can observe that the proposed BRR scheme can significantly reduce the amount of packet retransmissions, thereby conserving the network bandwidth. BRR can reduce the most packet retransmissions in the cases of locality (middle) and packet loss (skew right) in the simulations. The capability of such reduction depends on the locality of recovery requests. In particular, the lowest points of three cases of skew left, balance, and skew right in Fig. 6(a) locate at $T_{g A C K}=0.4 T, T_{g A C K}=0.5 T$, and $T_{g A C K}=0.6 T$, respectively, which are all close to the most locality of recovery requests (i.e., $0.5 T$ ). Similarly, in Fig. 6(b), the lowest points of three cases of skew left, balance, and skew right locate at $T_{g A C K}=0.6 T, T_{g A C K}=0.7 T$, and $T_{g A C K}=0.8 T$, respectively, which are all close to the most locality of recovery requests (i.e., $0.75 T$ ).

\section{CONCLUSIONS}

The DVB-H system provides the service of digital video broadcast to handheld devices in a mobile environment, but it suffers from a more serious packet loss problem. In this paper, we propose combining a DVB-H system with an IP-relay wireless network to support the recovery mechanism. Under the proposed architecture, we have addressed two important GPL and BDH problems, and developed a novel BBR scheme to solve these two problems. Our BRR scheme exploits the spatial and temporal locality of recovery requests, and can efficiently reduce duplicate submissions of recovery requests while merging retransmissions of lost packets. It is verified that our BRR scheme can significantly reduce the transmission amount of lost packets by simulations.

\section{ACKNOWLEDGMENTS}

Y.-C. Tseng's research is co-sponsored by MoE ATU Plan, by NSC grants 95-2221-E-009-058-MY3, 96-2218-E-009-004, 96-2219-E-007-008, 97-3114-E-009-001, 97-2221-E-009-142MY3, and 97-2218-E-009-026, by MOEA under grant 94-EC17-A-04-S1-044, by ITRI, Taiwan, and by III, Taiwan.

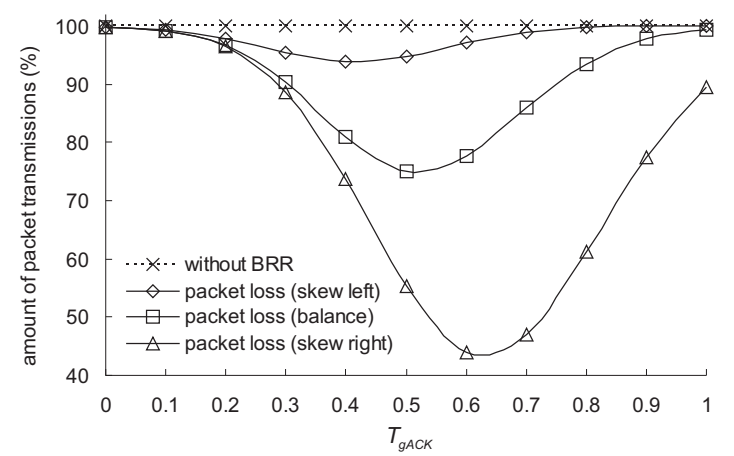

(a) locality (middle)

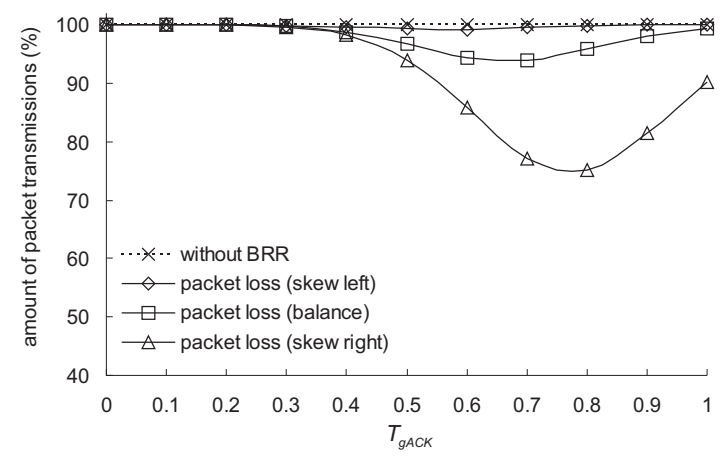

(b) locality (late)

Fig. 6: The transmission amount of lost packets under different distributions of $\mathcal{E}$ and $\mathcal{L}$.

\section{REFERENCES}

[1] G. Faria, J. A. Henriksson, E. Stare, and P. Talmola, "DVB-H: digital broadcast services to handheld devices," Proceedings of the IEEE, vol. 94, no. 1, pp. 194-209, 2006.

[2] G. Mastorakis, G. Kormentzas, and E. Pallis, "A fusion IP/DVB networking environment for providing always-on connectivity and tripleplay services to urban and rural areas," IEEE Network, vol. 21, no. 2, pp. 21-27, 2007.

[3] O. Eerenberg, A. Koppelaar, A. M. Stuivenwold, and P. H. N. de With, "IP-recovery in the DVB-H link layer for TV on mobile," in IEEE International Conference on Consumer Electronics, 2006, pp. 411-412.

[4] N. H. Vaidya and S. Hameed, "Scheduling data broadcast in asymmetric communication environments," Wireless Networks, no. 5, pp. 171-182, 1999.

[5] "IP datacast over DVB-H: architecture," ETSI, TS 102469 v1.1.1, May 2006.

[6] K. Lu, Y. Qian, H. H. Chen, and S. Fu, "WiMAX networks: from access to service platform," IEEE Network, vol. 22, no. 3, pp. 38-45, 2008.

[7] L. W. Chen, Y. C. Tseng, Y. C. Wang, D. W. Wang, and J. J. Wu, "Exploiting spectral reuse in routing, resource allocation, and scheduling for IEEE 802.16 mesh networks," IEEE Transactions on Vehicular Technology, vol. 58, no. 1, pp. 301-313, 2009.

[8] P. Pangalos, J. M. D. L. T. Velver, M. Dashti, A. Dashti, and H. Aghvami, "Confirming connectivity in interworked broadcast and mobile networks," IEEE Network, vol. 21, no. 2, pp. 13-20, 2007.

[9] R. Akester, "Reducing multicast collision loss for digital TV over 802.11 wireless networks," in WSEAS International Conference on Multimedia, Internet and Video Technologies, 2004.

[10] D. Gomez-Barquero, N. Cardona, A. Bria, and J. Zander, "Affordable mobile TV services in hybrid cellular and DVB-H systems," IEEE Network, vol. 21, no. 2, pp. 34-40, 2007.

[11] D. Gomez-Barquero and A. Bria, "Repair mechanisms for broadcast transmissions in hybrid cellular \& DVB-H systems," in IEEE International Symposium on Wireless Communication Systems, 2006, pp. 398402 . 